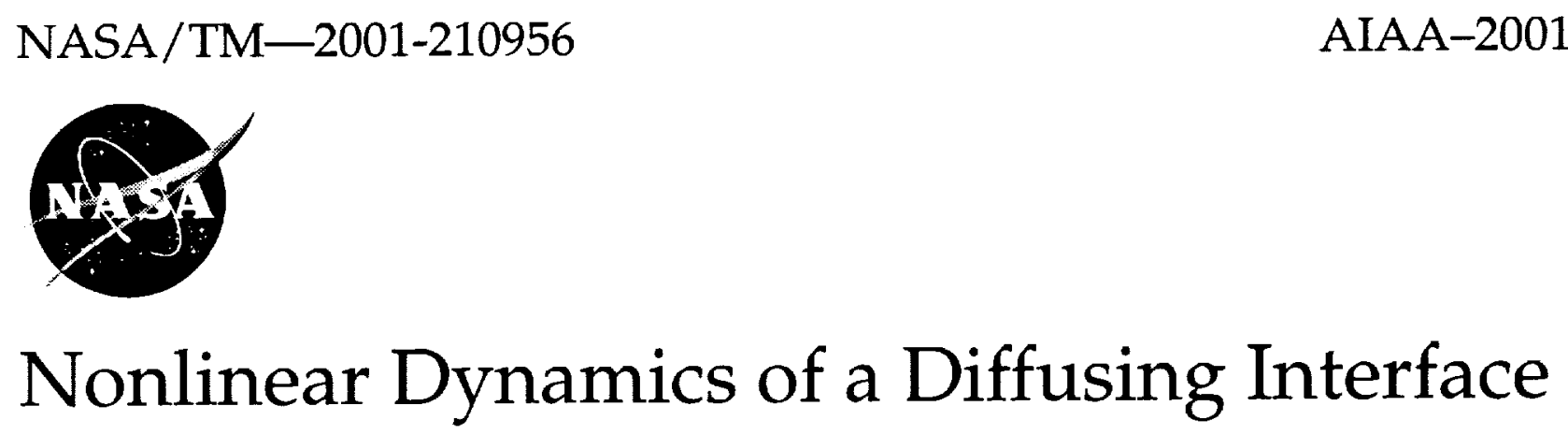

Walter M.B. Duval

Glenn Research Center, Cleveland, Ohio 
Since its founding, NASA has been dedicated to the advancement of aeronautics and space science. The NASA Scientific and Technical Information (STI) Program Office plays a key part in helping NASA maintain this important role.

The NASA STI Program Office is operated by Langley Research Center, the Lead Center for NASA's scientific and technical information. The NASA STI Program Office provides access to the NASA STI Database, the largest collection of aeronautical and space science STI in the world. The Program Office is also NASA's institutional mechanism for disseminating the results of its research and development activities. These results are published by NASA in the NASA STI Report Series, which includes the following report types:

- TECHNICAL PUBLICATION. Reports of completed research or a major significant phase of research that present the results of NASA programs and include extensive data or theoretical analysis. Includes compilations of significant scientific and technical data and information deemed to be of continuing reference value. NASA's counterpart of peerreviewed formal professional papers but has less stringent limitations on manuscript length and extent of graphic presentations.

- TECHNICAL MEMORANDUM. Scientific and technical findings that are preliminary or of specialized interest, e.g., quick release reports, working papers, and bibliographies that contain minimal annotation. Does not contain extensive analysis.

- CONTRACTOR REPORT. Scientific and technical findings by NASA-sponsored contractors and grantees.
- CONFERENCE PUBLICATION. Collected papers from scientific and technical conferences, symposia, seminars, or other meetings sponsored or cosponsored by NASA.

- SPECIAL PUBLICATION. Scientific, technical, or historical information from NASA programs, projects, and missions, often concerned with subjects having substantial public interest.

- TECHNICAL TRANSLATION. Englishlanguage translations of foreign scientific and technical material pertinent to NASA's mission.

Specialized services that complement the STI Program Office's diverse offerings include creating custom thesauri, building customized data bases, organizing and publishing research results ... even providing videos.

For more information about the NASA STI Program Office, see the following:

- Access the NASA STI Program Home Page at http://www.sti.nasa.gov

- E-mail your question via the Internet to help@sti.nasa.gov

- Fax your question to the NASA Access Help Desk at 301-621-0134

- Telephone the NASA Access Help Desk at 301-621-0390

- Write to: NASA Access Help Desk NASA Center for AeroSpace Information 7121 Standard Drive Hanover, MD 21076 
NASA/TM-2001-210956

AIAA-2001-1134

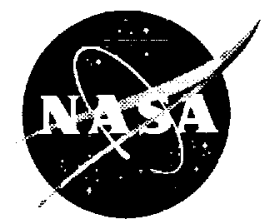

Nonlinear Dynamics of a Diffusing Interface

Walter M.B. Duval

Glenn Research Center, Cleveland, Ohio

Prepared for the

39th Aerospace Sciences Meeting and Exhibit

sponsored by the American Institute of Aeronautics and Astronautics

Reno, Nevada, January 8-11, 2001

National Aeronautics and

Space Administration

Glenn Research Center 


\section{Acknowledgments}

We thank code UG of NASA for their support under Grant No. NAG3-2443.

Available from

NASA Center for Aerospace Information 7121 Standard Drive

Hanover, MD 21076
National Technical Information Service 5285 Port Royal Road Springfield, VA 22100

Available electronically at http://gltrs.grc.nasa.gov/GLTRS 


\title{
NONLINEAR DYNAMICS OF A DIFFUSING INTERFACE
}

\author{
Walter M.B. Duval \\ National Aeronautics and Space Administration \\ Glenn Research Center \\ Cleveland, Ohio 44135
}

\begin{abstract}
Excitation of two miscible viscous liquids inside a bounded enclosure in a microgravity environment has shown the evolution of quasi-stationary waves of various modes for a range of parameters. We examine computationally the nonlinear dynamics of the system as the interface breakup and bifurcates to resonance structures typified by the Rayleigh-Taylor instability mechanism. Results show that when the mean steady field is much smaller than the amplitude of the sinusoidal excitation, the system behaves linearly, and growth of quasi-stationary waves occurs through the Kelvin-Helmholtz instability mechanism. However, as the amplitude of excitation increases, nonlinearity occurs through subharmonic bifurcation prior to broadband chaos.
\end{abstract}

\section{Introduction}

We examine computationally the nonlinear dynamics of flow fields, which destabilizes an initially stable sharp interface between two miscible liquids inside a cavity. The two fluids are subjected to oscillatory vibration in a microgravity environment, and serve as a model to understand effects of g-jitter. The destability of the interface between the two miscible liquids caused by bifurcation of the flow field serves as a model problem to study transport phenomena in a wide range of microgravity applications such as crystal growth of acousto-optic optoelectronic materials, protein and solution crystal growth. We consider a parametric space of relevance to microgravity applications.

The microgravity environment is particular
useful for transport phenomena involving nonhomogeneous bulk fluids due to minimization of buoyancy induced flows. However, vibration effects, which cause g-jitter, can serve as a source to induce convective flows. In order to quantify effects of g-jitter we designed an experiment to study effects of prescribed sinusoidal forcing on the stability of two miscible liquids ${ }^{1}$ The experimental results show that for the frequency band of interest $(0.1-10 \mathrm{~Hz})$ and disturbance amplitude $(0.2-$ 20 milli-g) the interface can indeed become unstable and generate quasi-stationary waves up to four modes. The instability of the interface was shown to cause by KelvinHelmholtz $(\mathrm{K}-\mathrm{H})$ instability ${ }^{2}$. The flow field destabilizes to an oscillating parallel shear flow and causes the interface to evolve like a vortex sheet. The parametric space for the experiment ranged from Stokes-Reynolds number (Res) of 0.002 to 0.5 . In this computational study, we extend Res to 43 .
We address the dynamical state of the flow field to gain insight on the predictability of the system. Of interest is the number of modes that can be generated for a quasi-stationary wave prior to its breakup. We examine the characteristic response of the system to various amplitude and frequency of excitation. We show that for Res $O(.1)$ the flow field responds isochronously to the input excitation, thus generates a limit cycle. The number of modes generated at the interface is correlated with the magnitude of Res. With increasing Res, the system becomes nonlinear and responds with harmonics of the input frequency. A subharmonic bifurcation occurs, similar to a two torus bifurcation, prior to broadband chaos. In the neighborhood of the chaotic state, there is breakup of the interface. The mechanism of the breakup is due to Rayleigh-Taylor instability.

In the following, we consider an infinite dimensional system as a dynamical model. We analyze the dynamical state of the system from its time history, pseudo and phase space trajectories, and power spectrum. The dynamical state of the system is correlated with the local bifurcation of the interface.

\section{Formulation}

The problem of interest is shown in Figure 1, two miscible liquids with distinct density meet initially at a planar interface. This realization has been shown to be possible in a microgravity environment. The two fluids are subjected to a body force parallel to its interface. The body force

$$
g(t)=n g_{0}+m g_{o}(\cos \omega t+\theta)
$$


consists of the steady background DC component (ngo), the excitation AC component with amplitude ( $\mathrm{mg}_{\mathrm{o}}$ ), circular frequency $\omega$, and phase angle $\theta$.

The dynamical model of the problem consists of the Navier-Stokes equations coupled with species continuity. For a square cavity $(\mathrm{H}=\mathrm{L})$ with characteristic length, time and velocity scales selected as,

$$
L_{c}=\sqrt{\bar{v} / \omega}, T=1 / \omega, U_{c}=\frac{\Delta \rho}{\bar{\rho}} \frac{m g_{o}}{\omega}
$$

The dimensionless incompressible Boussinesq equations in vorticity-stream function relationship can be expressed as,

$$
\begin{gathered}
\frac{\partial^{2} \psi}{\partial x^{2}}+\frac{\partial^{2} \psi}{\partial y^{2}}=-\zeta(3) \\
\frac{\partial \xi}{\partial t}+R e_{s}\left[u \frac{\partial \zeta}{\partial x}+v \frac{\partial \zeta}{\partial y}\right]=\left[\frac{\partial^{2} \zeta}{\partial x^{2}}+\frac{\partial^{2} \zeta}{\partial y^{2}}\right]+ \\
(A R+\cos (t+\theta)) \frac{\partial C^{*}}{\partial x} \\
\frac{\partial C^{*}}{\partial t}+R e_{s}\left[u \frac{\partial C^{*}}{\partial x}+v \frac{\partial C^{*}}{\partial y}\right]= \\
\frac{1}{S c}\left[\frac{\partial^{2} C^{*}}{\partial x^{2}}+\frac{\partial^{2} C^{*}}{\partial y^{2}}\right]
\end{gathered}
$$

The velocity components and vorticity are defined as,

$$
u=\frac{\partial \psi}{\partial y}, \quad \mathrm{v}=-\frac{\partial \psi}{\partial \mathrm{x}}, \quad \xi=\frac{\partial \mathrm{v}}{\partial \mathrm{x}}-\frac{\partial u}{\partial y}(6)
$$

The boundary conditions are no-slip along the wall boundaries $(\Gamma)$

$$
\vec{V} \bullet \vec{n}=0 \text { on } \Gamma(7)
$$

and the condition of impermeability normal $(\bar{n})$ to the boundary,

$$
\nabla C^{*} \bullet \vec{n}=0 \text { on } \Gamma
$$

Since we are considering an initial value problem, the concentration field has prescribed values of 1 and 0 for the left and right fluids respectively, with a value of 0.5 for the interface,

$$
C^{*}(x, y, z, 0)=\left\{\begin{array}{lc}
1.0 & 0 \leq x \leq L / 2 \\
0.5 & x=L / 2 \\
0 & L / 2 \leq x \leq L
\end{array}\right.
$$

The concentration field equation is normalized with $\mathrm{C}^{*}=$ $\left(C-C_{B}\right) /\left(C_{A}-C_{B}\right)$. The parametric space $(\Lambda)$ of the above set of equations

$$
\Lambda=A(\operatorname{Res}, A R, S c, \theta)(10)
$$

consists of the Stokes-Reynolds (Res) number, the amplitude ratio (AR), the Schmidt number $(\mathrm{Sc})$, and the phase angle $\theta$ defined as,

$$
\operatorname{Re}_{s}=\frac{\Delta \rho m g_{o}}{\bar{\rho} \bar{v}^{1 / 2} \omega^{3 / 2}} \quad, \quad A R=\frac{n}{m}, S c=\frac{\bar{v}}{D_{A B}}
$$

For the parameters of interest, the Schmidt number is kept constant as well as the phase angle. Thus the flow field as well as the dynamical evolution of the interface can be expressed as a co-dimension two problem,

$$
\vec{V}=\vec{V}(x, y, t ; \operatorname{Re} s, A R)
$$

We used finite difference to solve the coupled set of equations (3-5). Since we are interested in the asymptotic dynamics of the system, we used an explicit scheme with a third order Adams-Bashforth technique for time discretization. The Flux Corrected Transport algorithm is used to resolve the sharp discontinuity of the interface $^{3}$. A $90 \times 90$ grid size is used for spatial resolution and found to be adequate over the parametric range of interest. We mostly varied Res for our parametric studies, however, we found that the results are sensitive to the magnitude of AR.

\section{Discussion and Computational Results}

The parametric space considered consists of variation of the amplitude of excitation $\left(\mathrm{mg}_{\mathrm{o}}\right)$, input frequency $(\omega)$, while keeping the background $D C$ acceleration $\left(\mathrm{ng}_{0}=10^{-6} \mathrm{~g}_{0}, \mathrm{~g}_{0}=980 \mathrm{~cm} / \mathrm{sec}^{2}\right.$ ) and phase angle $(\theta=0)$ fixed. The geometric length scales $(\mathrm{H}=\mathrm{L}=5 \mathrm{~cm})$ and properties of the fluid $(\mathrm{Sc}=1079)$ are fixed. The range of parameters considered is shown in Table 1. 
Table 1 - Range of Parameters, $\mathrm{ng}_{0}=10^{-6} \mathrm{~g}_{\mathrm{o}}$

\begin{tabular}{|c|c|c|}
\hline $\begin{array}{c}f \\
(\mathrm{~Hz})\end{array}$ & Res & AR \\
\hline 0.5 & 0.1908 & $2.5 \times 10^{-4}$ \\
\hline 0.5 & 0.2386 & $2.0 \times 10^{-4}$ \\
\hline 0.5 & 0.2863 & $1.67 \times 10^{-4}$ \\
\hline 0.5 & 0.3818 & $1.25 \times 10^{-4}$ \\
\hline 0.5 & 0.4772 & $1.0 \times 10^{-4}$ \\
\hline 0.5 & 0.7158 & $6.67 \times 10^{-5}$ \\
\hline 0.5 & 0.95 & $5.0 \times 10^{-5}$ \\
\hline 0.33 & 1.78 & $2.50 \times 10^{-5}$ \\
\hline 0.25 & 2.69 & $2.50 \times 10^{-5}$ \\
\hline 0.5 & 2.85 & $1.67 \times 10^{-5}$ \\
\hline 0.5 & 3.80 & $1.25 \times 10^{-5}$ \\
\hline 0.5 & 4.75 & $1.0 \times 10^{-5}$ \\
\hline 0.5 & 5.70 & $8.33 \times 10^{-5}$ \\
\hline 0.5 & 6.67 & $7.14 \times 10^{-6}$ \\
\hline 0.5 & 7.60 & $6.25 \times 10^{-6}$ \\
\hline 0.5 & 8.55 & $5.56 \times 10^{-6}$ \\
\hline 0.5 & 9.5 & $5.0 \times 10^{-6}$ \\
\hline 0.5 & 10.45 & $4.55 \times 10^{-6}$ \\
\hline$:$ & $:$ & $:$ \\
\hline 0.5 & 42.75 & $1.11 \times 10^{-6}$ \\
\hline
\end{tabular}

The metric used to quantify the response of the interface to various excitation input is its wavelength $(\lambda)$ which is a function of Res and $A R$,

$$
\lambda=\lambda(\operatorname{Res}, \mathrm{AR})
$$

Even the co-dimension two bifurcation problem is a formidable computational task because the system loses and gains stability over a dense parametric set similar to the logistic map model ${ }^{4}$. Even though all the fine details of the bifurcation sequence are not computed, the range of parameters in Table I gives a broad glimpse on typical scenarios. Over most of the parametric space, we varied $\mathrm{mg}_{0}$, a narrow range of $\omega$ was considered.

\section{Effect of Flow Field on Dynamics of Interface}

In order to correlate the dynamical equilibrium of the flow field in phase space with the interface response, we show in Figure 2 the local bifurcation of the quasistationary wave as a function of Res. The wavelength of the $\mathrm{q}-\mathrm{s}$ wave decreases, or the number of modes increases, as Res increases. Breakup of the interface occurs in the neighborhood of $\operatorname{Res}=8.55$ for which q-s waves no longer exist. The number of modes is dictated by the number of bends in the interface, an $S$ shape has two modes. Transition from two to four mode $q$-s wave occurs for
Res $>0.29$. Typical interface response of four mode $q-s$ wave is shown for Res=0.72; the four mode response extends up to Res $=1.78$. The mode number at the interface is sensitive to the threshold of $A R$, for example at Res $=0.34$ and $A R=5 \times 10^{-5}$ a four mode structure can be suppressed by increasing AR to $1.25 \times 10^{-4}$, only two modes result. There is a transition from 4 to 8 primary modes for Res $=2.85$; note 3 secondary modes occur with very small wavelength at the top and bottom boundaries. This bifurcation occurs in the neighborhood of Res=1.9.

The mechanism which generates q-s waves for Res $O(2.85)$ is basically the Kelvin-Helmholtz $(\mathrm{K}-\mathrm{H})$ instability. A row of parallel vortices is generated along the interface. These vortices serve as rotors, which stretch and fold the interface. As Res increases, Res $\mathrm{O}(4.75)$, there occur secondary waves riding on the primary modes on the interface. The K-H instability mechanism manifest itself perpendicular to the interface to form vortex rows. The interface no longer forms $q-s$ waves, rather stretches and folds continuously to generate roll-up structures as typified by the Rayleigh-Taylor instability ${ }^{5}$. Only the initial state of the interface structure is shown, the flow field becomes quite complex with vortices occupying the entire cavity. The state of the flow field is chaotic as will later be shown.

The wavelength $(\lambda)$ of the interface, up to the breakup, decreases asymptotically as Res increases, shown in Figure 3. The wavelength is estimated from its average value at the center of the cavity. The dependence of $\lambda$ on Res follows a power law

$$
\lambda \propto \operatorname{CRes}^{-n}(14)
$$

$\mathrm{C}$ is a constant and $\mathrm{n}$ is less than 1 ; the decrease in wavelength is similar to a turbulent cascade. In contrast the maximum magnitude of the velocity $|V|_{\max }$, shown in Figure 4, increases with Res and also follows a power law in which $\mathrm{n}$ is greater than 1 ,

$$
V_{\max } \propto \operatorname{CRes}^{n}
$$

The functional relationship of $\lambda$ and $V_{\max }$ on Res illustrate the global trends. Much more details would be needed to show the bifurcation details as found for example in the logistic map.

\section{Dyamical State of the Flow Field}

We characterize the dynamical state of the system from the time history of the asymptotic dynamics of the flow field. The limit set of the time history is obtained 
from both the pseudo and phase space trajectories. For a given frequency and amplitude of excitation, the response of the system is obtained from its power spectrum. The estimated power spectrum for the $\mathrm{v}$ component of the velocity field is calculated from,

$$
\widetilde{P}_{V}(f)=\frac{1}{R}\left|\int_{0}^{R} V(t) e^{-i 2 \pi f t} d t\right|^{2}
$$

in which $\mathrm{R}$ is the finite duration of the time interval. The smooth spectral estimate $\mathrm{P}_{\mathrm{V}}(f)$ is obtained using a Hanning window for filtering, and a convolution relation as,

$$
P_{v}(f)=W_{s}(f) * \tilde{P}_{v}(f)
$$

$\mathrm{W}_{\mathrm{s}}(f)$ is the spectral window for filtering. The power spectrum is estimated using the Cooley-Tukey algorithm, which employs the use of Fast Fourier Transforms ${ }^{6.7}$. The dynamics of the flow field is evaluated from a representative point of the flow field. For each data set we kept 10,000 points.

We now analyze the dynamical state of the flow field, which generates the q-s waves shown in Figure 2. For low Res $=0.19$ which yields a two-mode q-s wave, the flow field oscillate isochronously, see Figure 5a, with the input frequency $\mathrm{f}=0.5 \mathrm{~Hz}$. The power spectrum shows that the $\mathrm{v}$ component of the flow field responds with a frequency of $0.5 \mathrm{~Hz}$, the same scenario was found for the u component. The pseudo-phase space trajectory, found by lagging the velocity with a constant $\mathrm{k}$, of the $\mathrm{v}$ component indicates that the dynamics approach a limit cycle attractor for the selected location $(0.33,0.56)$ in the flow field. This indicates that for the given parametric input to the system, its future behavior will always tend to oscillate in phase with the input frequency.

When nonlinearity becomes important, Figure $5 \mathrm{~b}$ Res $=0.48$, harmonics of the fundamental frequency appear. While the u component of the velocity field is periodic, as shown above, the $\mathrm{v}$ component of the velocity shows a decrease in amplitude with every oscillation. The combination of the signal is shown in the phase space trajectory, which shows a nonchaotic attractor. This attractor is a smooth surface with the attributes of stretching and folding in phase-space.

With increasing nonlinearity in the system, Figure 6 Res $=0.72$, the flow field responds with a modulated amplitude signal in the $\mathrm{v}$ component. Such modulation gives rise to nonchaotic attractors as evidence from the pseudo-phase space trajectory of $v(t+k)$; this attractor is similar to the Rossler funnel ${ }^{8}$. The flow field responds with harmonics $(1.0,1.5 \mathrm{~Hz})$ of the fundamental input frequency of $0.5 \mathrm{~Hz}$; the $1.5 \mathrm{~Hz}$ component is very minute and is barely discemible. The gradual decrease in the amplitude of the u component of the velocity field gives rise to the dense limit cycle attractor. The combination of the signal of the $u$ and $v$ component is shown in the $(u, v)$ phase-space trajectory, which shows the evolution of a nonchaotic attractor. It is known that strange nonchaotic attractors exist for quasi-periodically forced systems ${ }^{9.10,11}$, however they are not smooth manifolds in phase space. Even though the attractors shown in Figure 6 are nonchaotic, they are not strange. This study gives insight into parametric regions for which an infinite-dimensional system may display these so-called strange nonchaotic attractors.

A rational subharmonic bifurcation is shown in Figure 7. When Res is increased to 1.78 for an input frequency of $0.33 \mathrm{~Hz}$, both the $u$ and $v$ component of the velocity field show harmonic response of the fundamental frequency. A complex nonchaotic attractor illustrating stretching and folding arises in phase-space. A subharmonic bifurcation occurred in the neighborhood of Res $=2.69, A R=2.5 \times 10^{-5}$. In contrast to the above cases, both the $\mathrm{u}$ and $\mathrm{v}$ components show response at one-half the input frequency of $0.25 \mathrm{~Hz}$ as well as higher harmonics. The response frequency of the system is phase-locked to the input frequency due to the rational relationship of the frequency ratio. Note that a subharmonic attractor is generated in phase space with a contraction of volume, since the system is dissipative; this reduces the complexity of the attractor in comparison to the lower values of Res. The amplitude ratio AR plays a significant role in the bifurcation sequence, for the same Res value of 2.69 if AR increases to $1.7 \times 10^{-5}$ then the subharmonic bifurcation dissapears, only harmonics of the fundamental frequency occurs.

The dynamics of the system for which it transitions from $\mathrm{q}-\mathrm{s}$ waves to tendril structures and breakup is shown in Figure 8. Corresponding to the tendril structure, Res $=4.75$, is an irrational frequency component of the fundamental input frequency shown in the power spectrum. Similar to $\operatorname{Res}=2.69$, there is a subharmonic attractor with contraction of volume in phase space. This corresponds to the two-torus bifurcation as proposed by Newhouse, Ruelle, and Takens ${ }^{12}$. The mechanism of K-H instability which causes secondary waves on the interface gives rise to the complexity of the flow field which generates these waves. In the neighborhood of the breakup region, $\operatorname{Res}=7.6$, there is a resulting broadband power spectrum distribution which shows that the system responds to all the frequency components within the interval of $1.0 \mathrm{~Hz}$. Since the dynamics of the system 
changes after breakup, we partitioned the signal of the $\mathrm{v}$ component into two intervals. Contraction of volume occurs in phase space for the first interval. The second interval from 160 to 400 secs shows the asymptotic dynamics of the system which yields a chaotic attractor. This attractor has the attributes of folding and stretching which typifies unpredictability. The results for Res in the interval of 7.6 to 42.75 show that there is a sequence of window for which the system gains stability to become periodic and loses stability to become chaotic similar to the logistic map.

\section{Summary and Conclusions}

We considered the nonlinear dynamics of flow fields, which destabilizes an interface through the KelvinHelmholtz instability mechanism to cause quasi-stationary waves. The wavelength decreases asymptotically as Res increases up to the breakup region; the decrease in wavelength is similar to a turbulent cascade). We show that when the system becomes nonlinear there is a transition from two to four mode q-s waves which gives rise to a host of nonchaotic attractors due to harmonics of the fundamental frequency in the response signal of the velocity component. Bifurcation of the system from q-s waves to tendril structures introduces a rational subharmonic bifurcation. Chaos occurs through an irrational subharmonic bifurcation, which corresponds to a two-torus bifurcation; the interface breaks up and the Raleigh-Taylor type of instability is manifested. Though Res was not varied continuously to capture all the bifurcation details, the subset of the parametric space considered lead insight into the dynamical behavior of the system.

\section{$\underline{\text { References }}$}

1) Duval, W.M.B., Tryggvason, B.V., " Effects of GJitter on Interfacial Dynamics of Two Miscible Liquids: Application of MIM," NASA/TM-2000209789, March 2000.

2) Duval, W.M.B., "Sensitivity of a Wave Structure to Initial Conditions," $38^{\text {th }}$ Aerospace Sciences Meeting \& Exhibit, AIAA-2000-1051 Paper, Jan. 10-13, 2000 .

3) Boris, J.P., and Book, D.L., "Flux-Corrected Transport I; SHASTA-A Fluid Transport Algorithm That Works," J. Comp. Phys., Vol. 11, pp. 38-69, 1973.
4) Baker, G.L., Gollub, J.P. Chaotic Dynamics. Cambridge University Press, 1990.

5) Sharp, D.H., "An Overview of Rayleigh-Taylor Instability, Physica 12D, pp. 3-8, 1984.

6) Cooley, J.W., Tukey, J.W., "An algorithm for the Machine Calculation of Complex Fourier Series," Mathematics of Computations, Vol. 19, pp. 297-30I, 1965.

7) Brigham, E.O. The Fast Fourier Transform. PrenticeHall, Englewood Cliffs, New Jersey, 1974.

8) Rossler, O., "Different Types of Chaos in Two Simple Differential Equations," Zeitschr. Naturforsch., Vol. 31a, pp. 1664-1670, 1980.

9) Romeiras, F.J., Bondeson, A., Ott, E., Antonsen, T.M., Grebogi, C., " Quasiperiodically Forced Dynamical Systems with Strange Nonchaotic Attractors," Physica 12 D, pp. 277-294, 1987.

10) Romeiras, F.J., Ott, E., "Strange Nonchaotic Attractors of the Damped Pendulum with Quasiperiodic Forcing," Phys. Rev. A, Vol. 35, No. 10, pp. 4404-4413, 1987.

11) Kapitaniak, T., Wojewoda, J., "Strange NonChaotic Attractors of a Quasi-Periodically Forced Van Der Pol's Oscillator," J. of Sound and Vibration, Vol. 138(1), pp. 162-I69, 1990.

12) Newhouse, S., Ruelle, D., Takens, F., "Occurrence of Strange Axiom A Attractors Near Quasi-Periodic Flows on $T^{m}, m \geq 3$," Communication in Mathematical Physics, Vol. 64, pp. 35-40, 1978. 


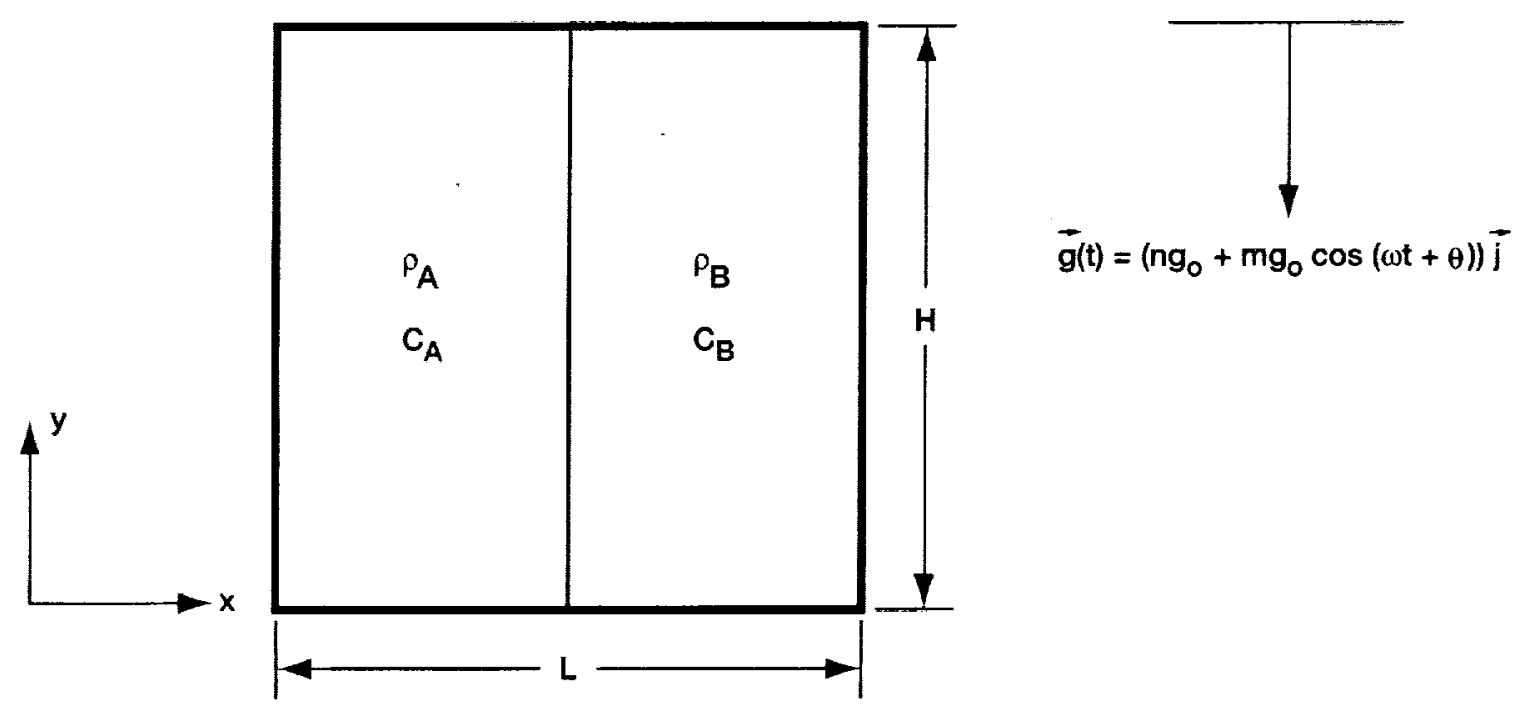

Figure 1-Physical description of two fluids in contact at an interface. 
$\mathrm{t}=160 \mathrm{~s}, \mathrm{mg}_{\mathrm{o}}=15 \mathrm{mg}, \mathrm{AR}=6.67 \times 10^{-5}, \mathrm{Re}_{\mathrm{s}}=0.72, \mathrm{~V}_{\max }=0.049 \mathrm{~cm} / \mathrm{sec}$
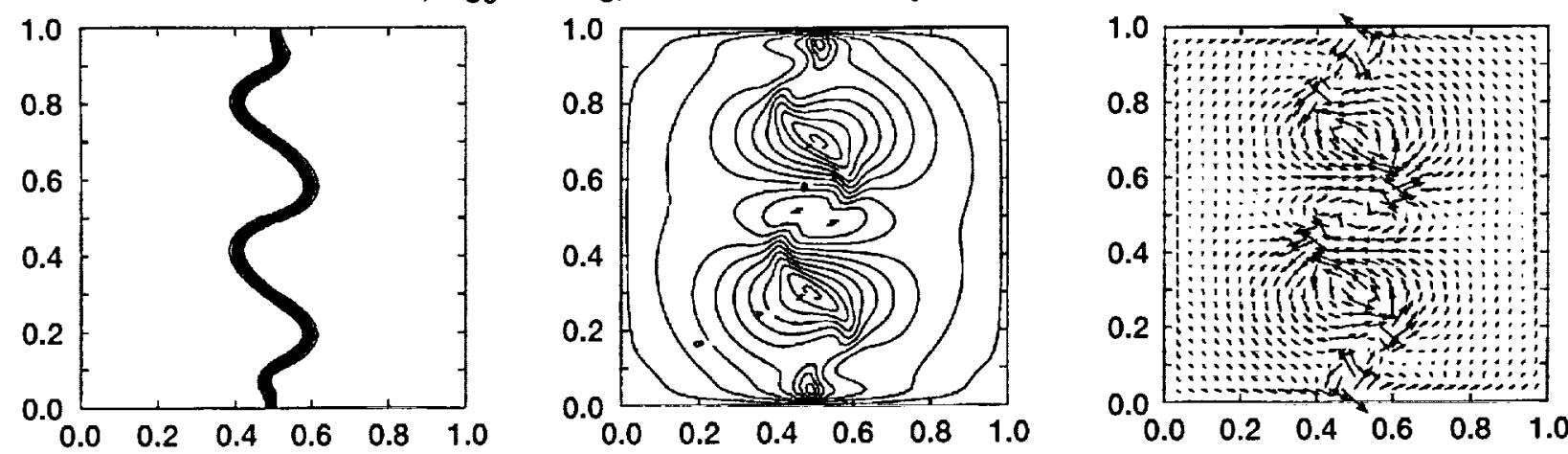

$\mathrm{t}=20 \mathrm{~s}, \mathrm{mg}_{\mathrm{o}}=60 \mathrm{mg}, \mathrm{AR}=1.67 \times 10^{-5}, \mathrm{Re}_{\mathrm{s}}=2.85, \mathrm{~V}_{\max }=0.18 \mathrm{~cm} / \mathrm{sec}$
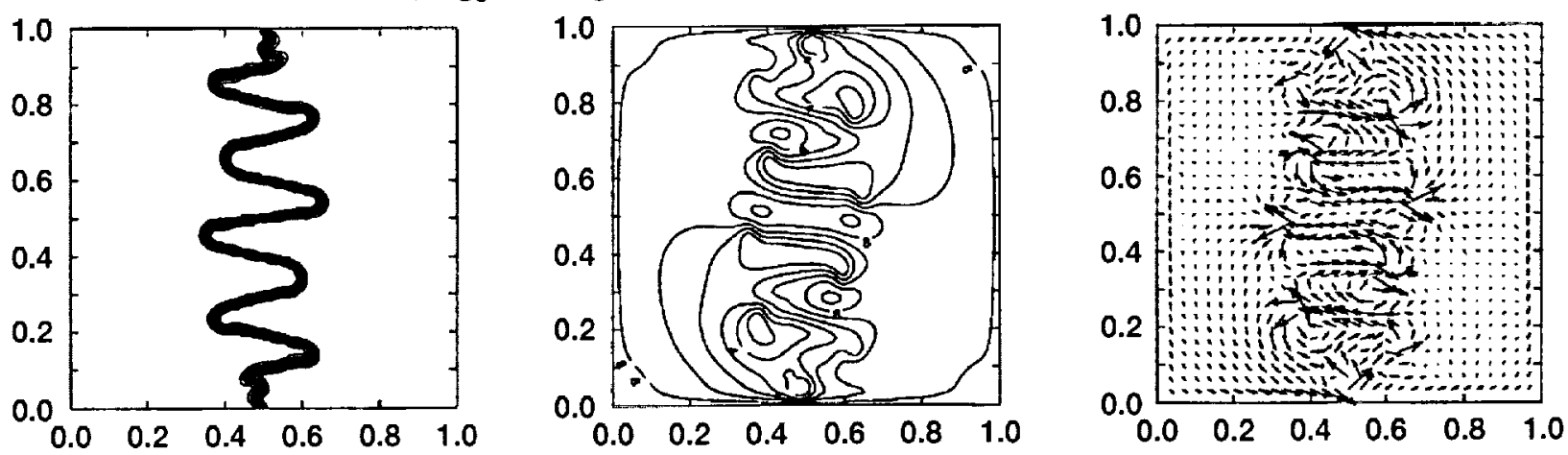

$t=18 \mathrm{~s}, \mathrm{mg}_{0}=100 \mathrm{mg}, \mathrm{AR}=1 \times 10^{-5}, R_{\mathrm{s}}=4.75, \mathrm{~V}_{\max }=0.29 \mathrm{~cm} / \mathrm{sec}$
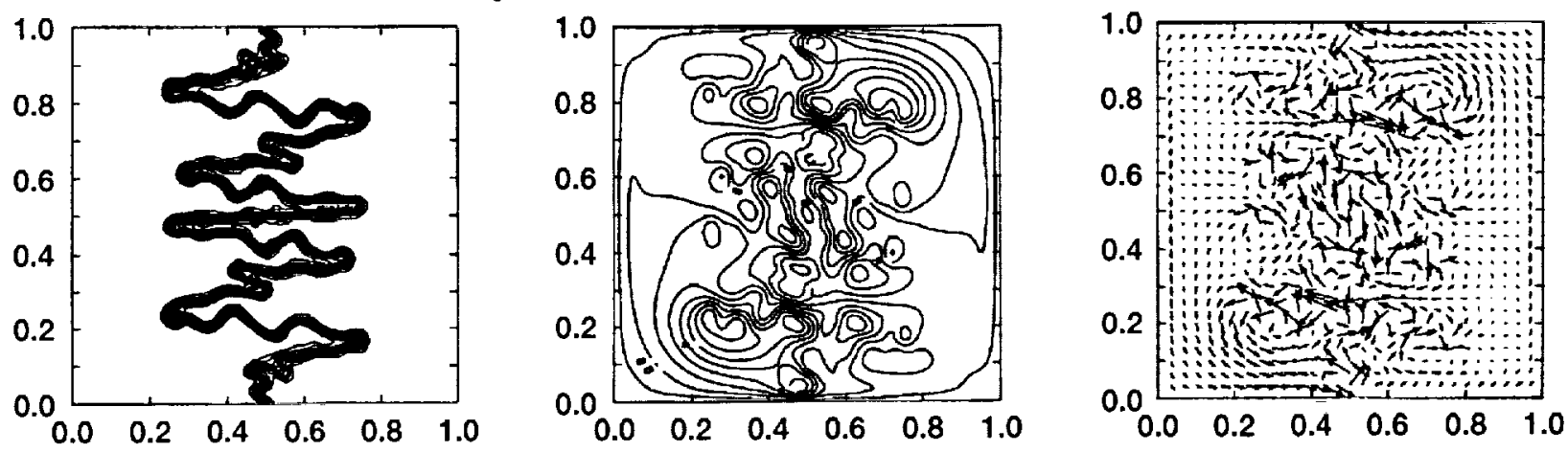

$\mathrm{t}=6 \mathrm{~s}, \mathrm{mg}_{\mathrm{o}}=180 \mathrm{mg}, \mathrm{AR}=5.56 \times 10^{-6}, \mathrm{Re}_{\mathrm{s}}=8.55, \mathrm{~V}_{\max }=0.58 \mathrm{~cm} / \mathrm{sec}$
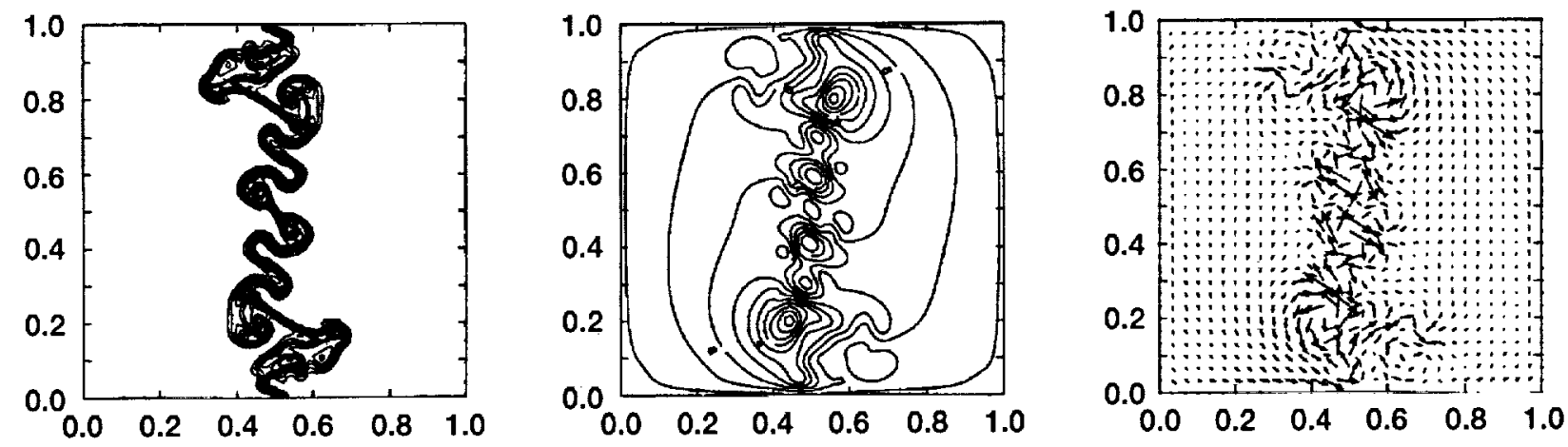

Figure 2.-Bifurcation of quasi-stationary waves showing increasing density of modes up to breakup $\left(\operatorname{Re}_{\mathrm{s}}=8.55\right), \mathrm{f}=0.5 \mathrm{~Hz}, \mathrm{ng}_{\mathrm{o}}=10^{-6} \mathrm{~g}_{\mathrm{o}}$. 


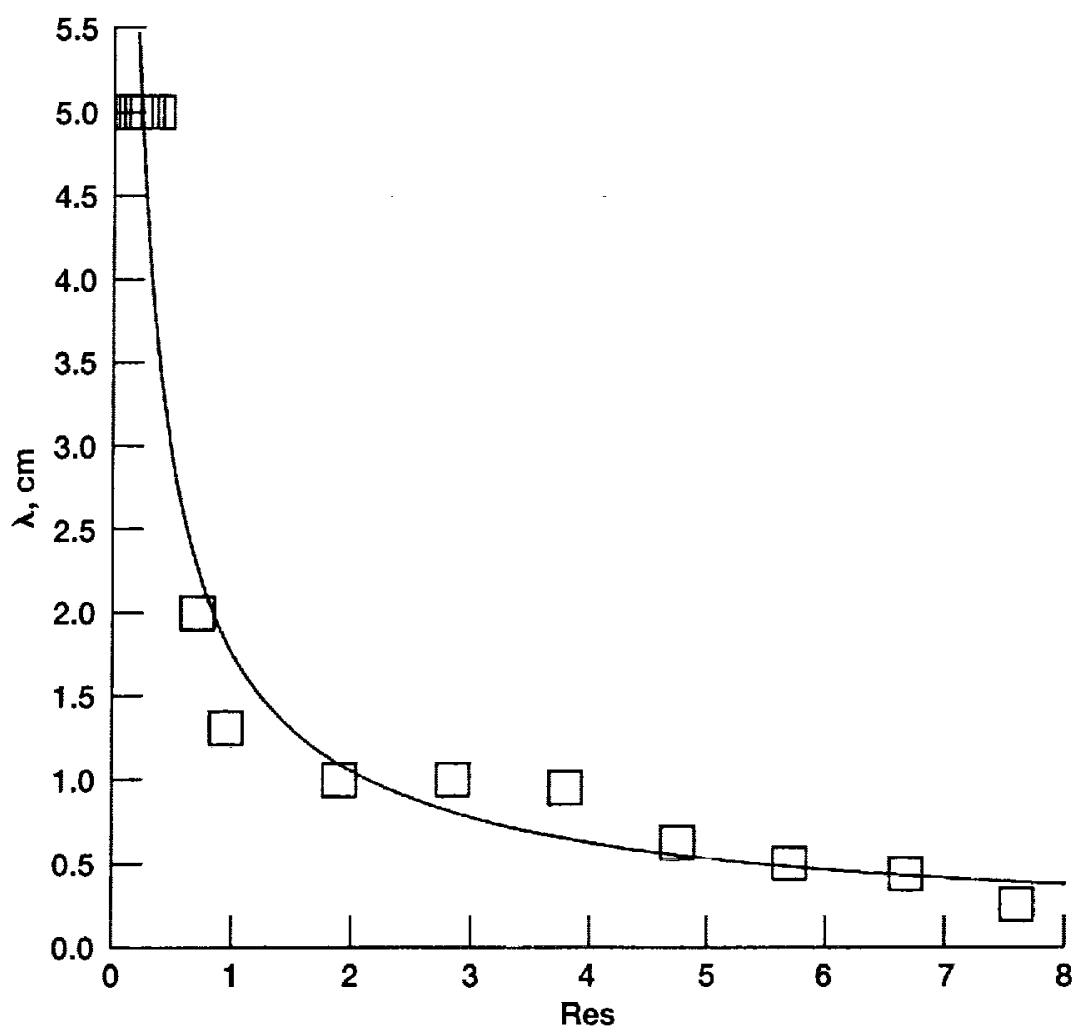

Figure 3.-Wavelength $(\lambda)$ as a function of Stokes-Reynolds number.

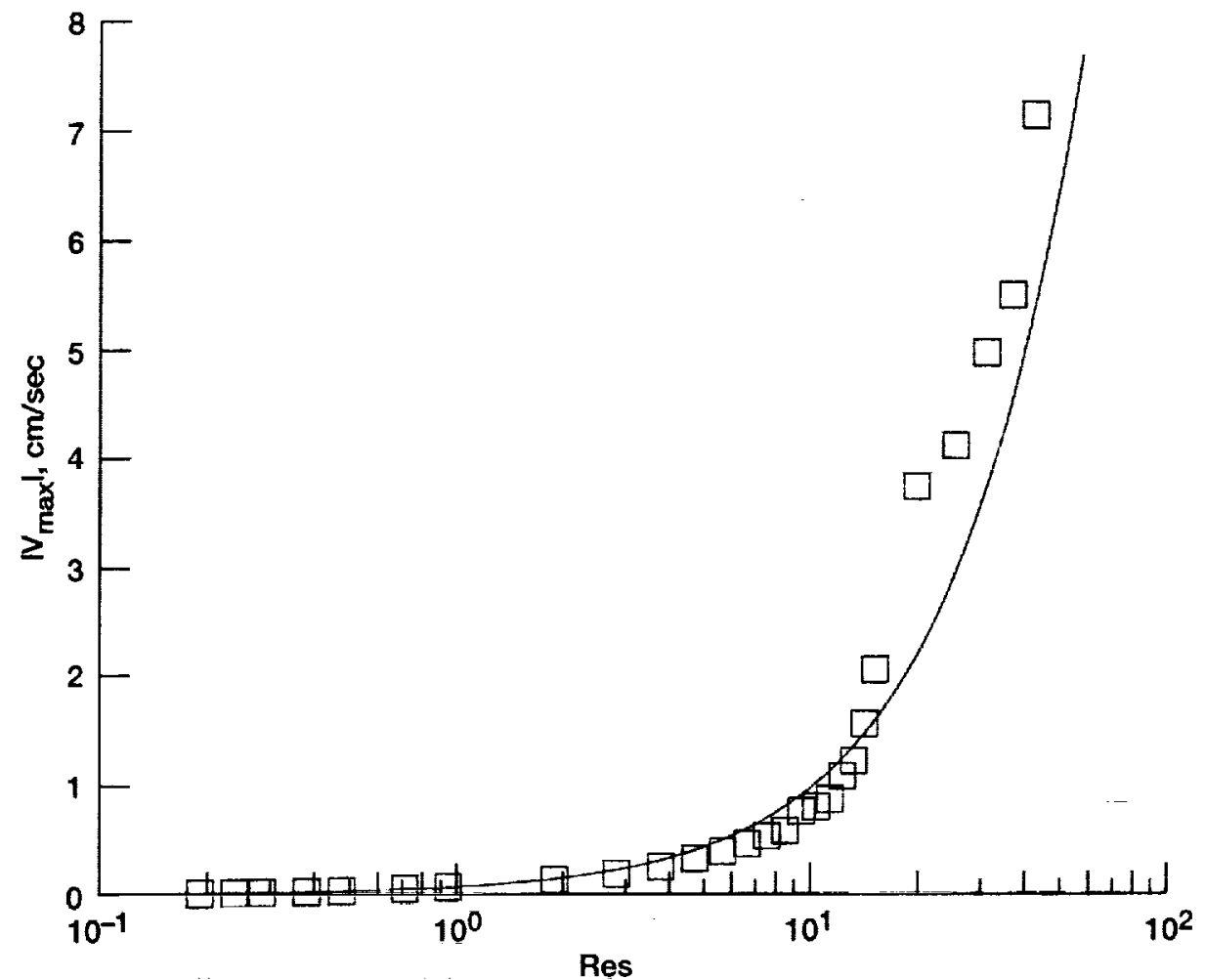

Figure 4.-Maximum velocity as a function of Stokes-Reynolds (Res) number. 

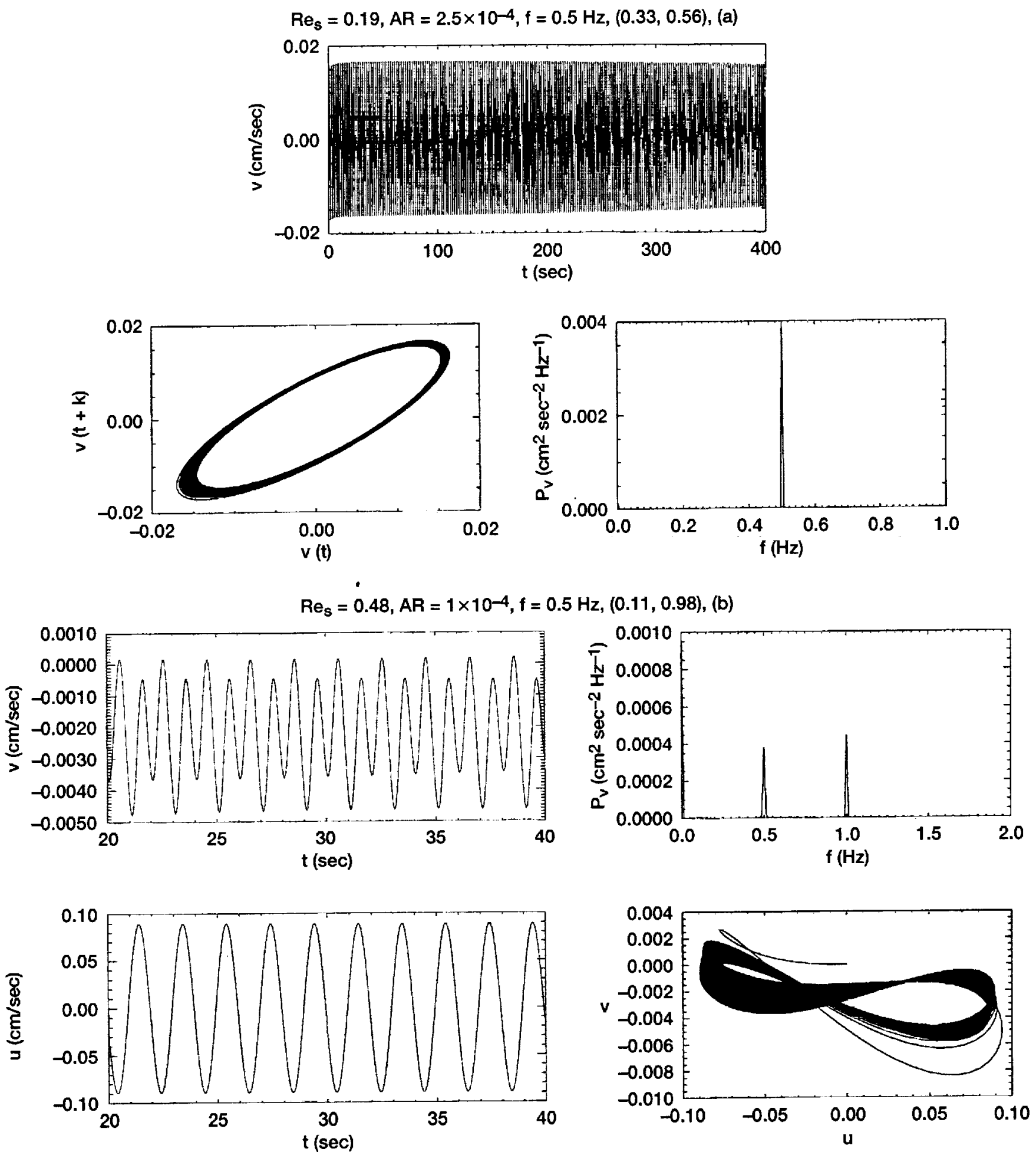

Figure 5.-Limit cycle $\left(R e_{s}=0.19\right)$ and nonchaotic attractor $\left(R e_{s}=0.48\right)$ due to aperiodic component of velocity field. 

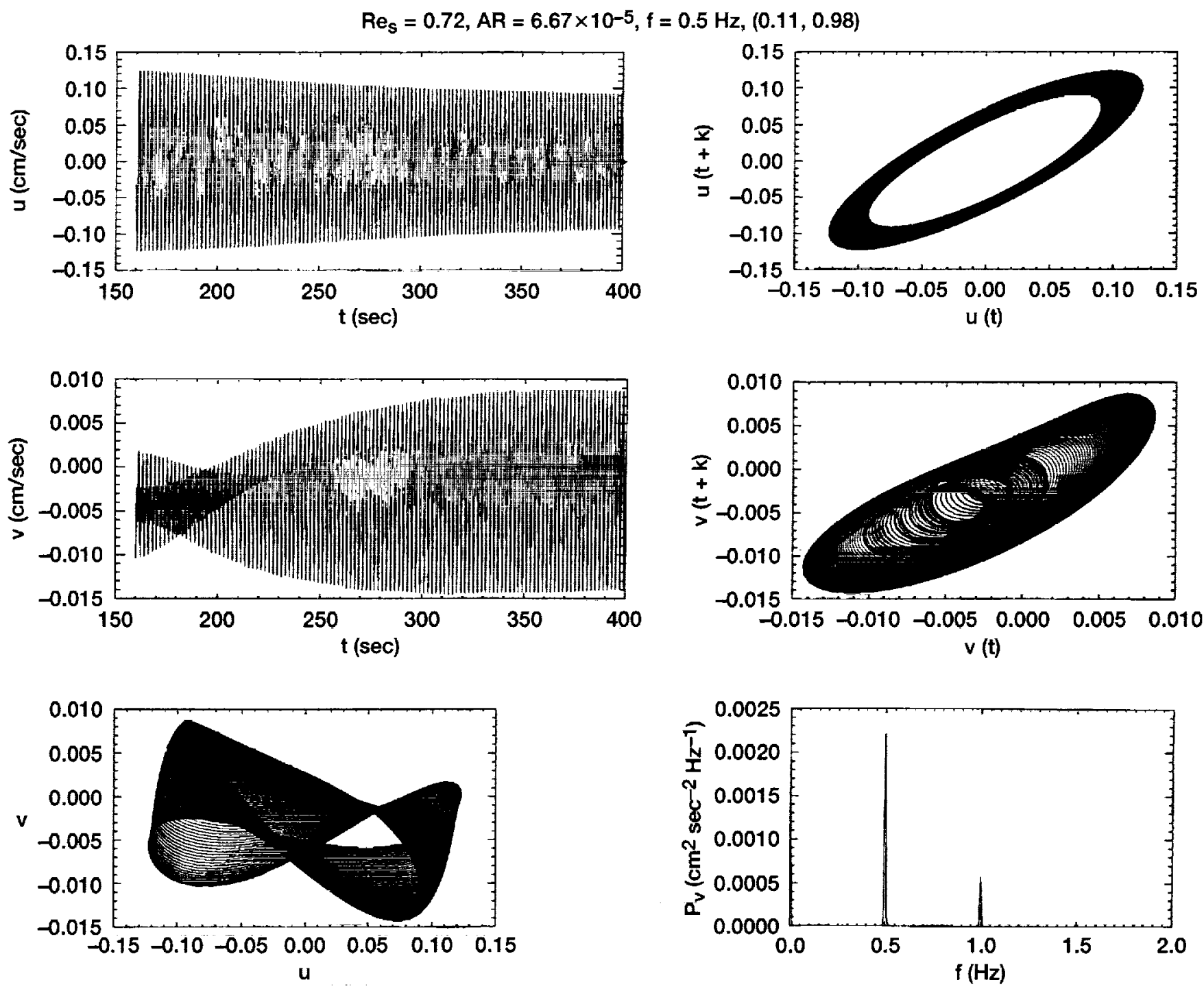

Figure 6.-Nonchaotic attractor due to periodic and amplitude modulated components of velocity field. 

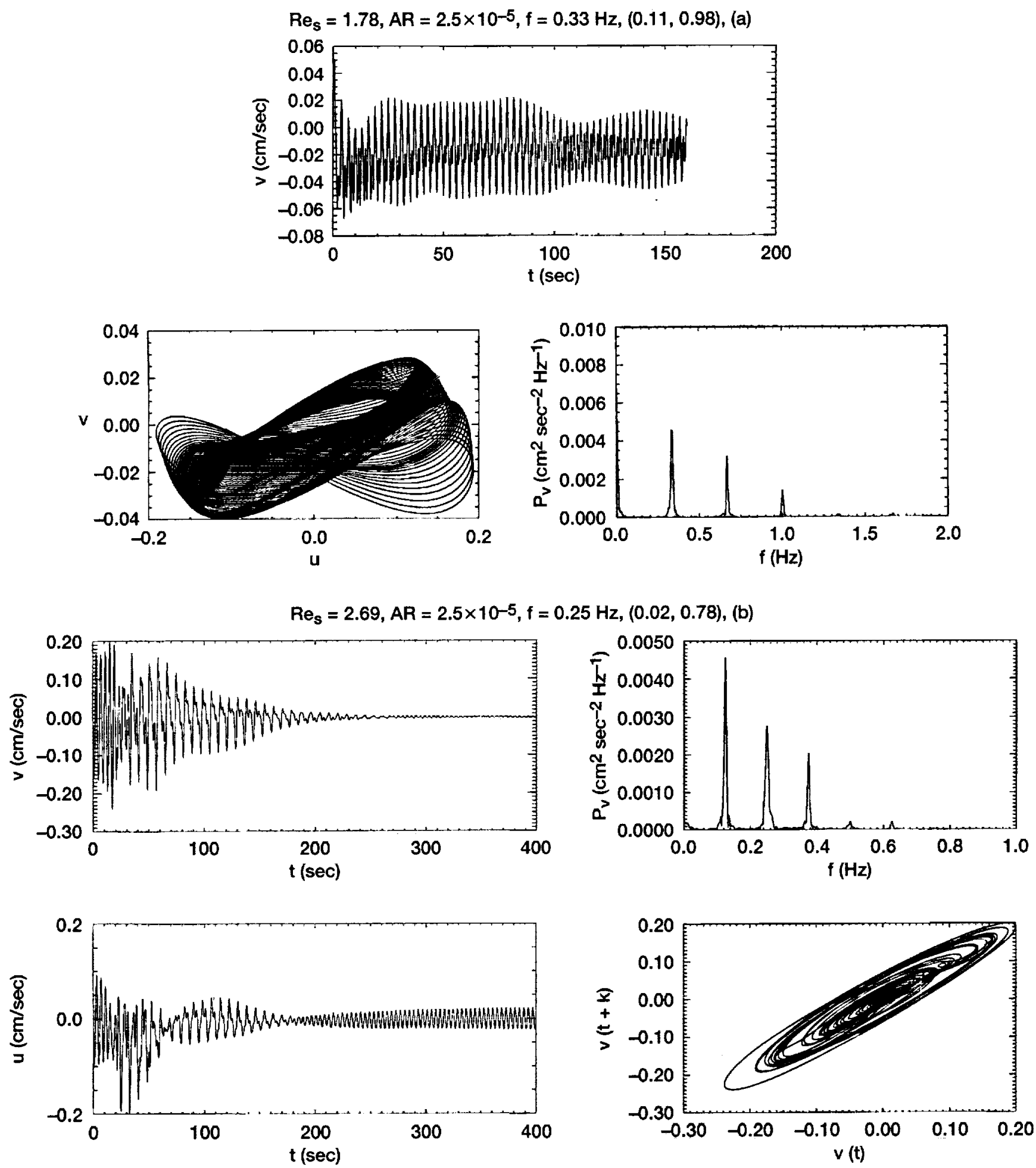

Figure 7.-Rational subharmonic bifurcation of velocity field. 

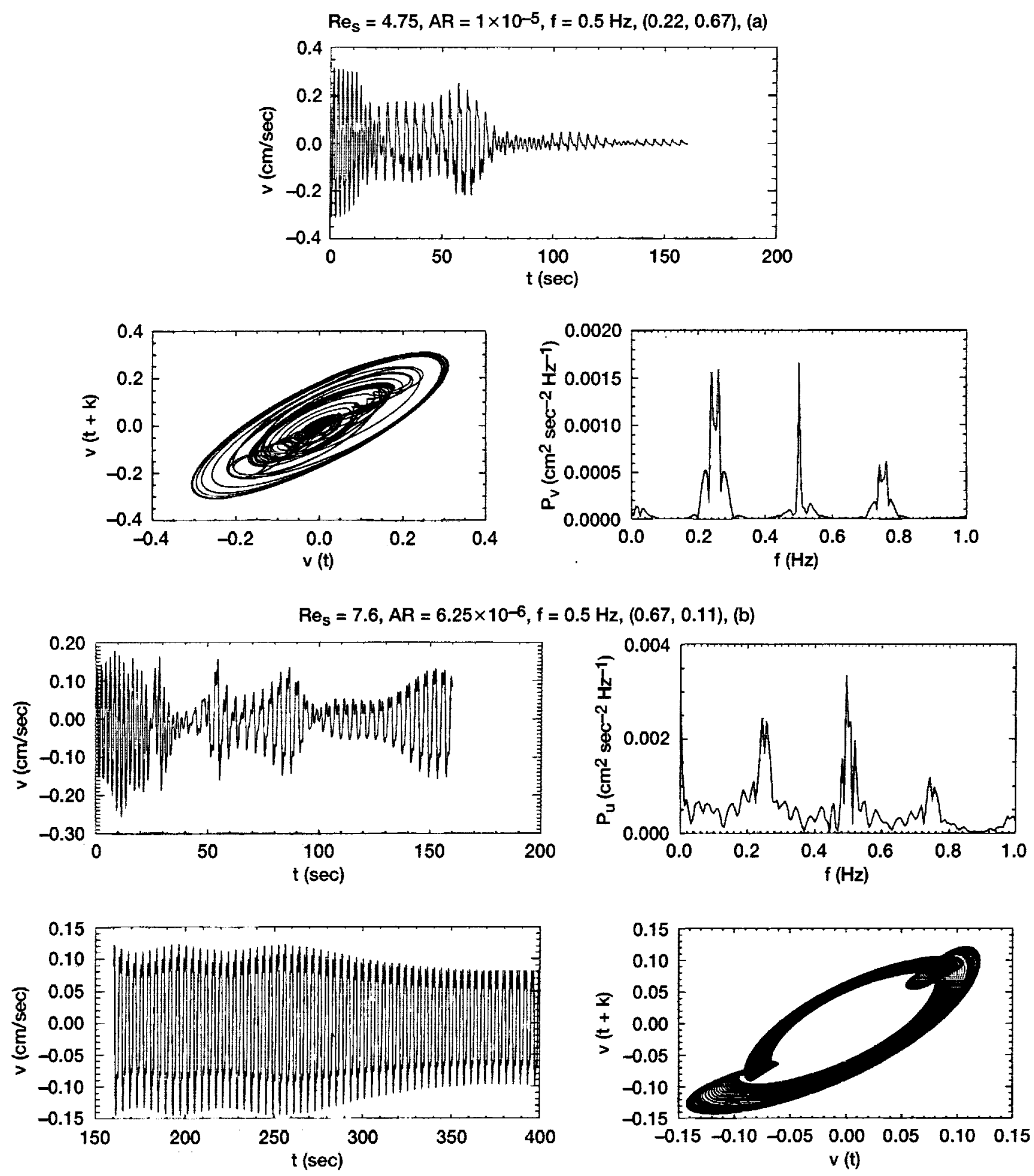

Figure 8.-Transition to chaos via irrational subharmonic bifurcation. 
- 
Public reporting burden for this collection of information is estimated to average 1 hour per response, including the time tor reviewing instructions, searching existing data sources, gathering and maintaining the data needed, and completing and reviewing the collection of information. Send comments regarding this burden estimate or any other aspect of this collection of information, including suggestions for reducing this burden, to Washington Headquarters Services, Directorate for Information Operations and Reports, 1215 Jefferson Davis Highway, Suite 1204, Artington, VA 22202-4302, and to the Office of Management and Budget, Paperwork Reduction Project (0704-0188), Washington, DC 20503.

\begin{tabular}{|l|c|c|}
\hline 1. AGENCY USE ONLY (Leave blank) & $\begin{array}{c}\text { 2. REPORT DATE } \\
\text { October } 2001\end{array}$ & $\begin{array}{c}\text { 3. REPORT TYPE AND DATES COVEAED } \\
\text { Technical Memorandum }\end{array}$ \\
\hline
\end{tabular}

\section{TITLE AND SUBTITLE}

Nonlinear Dynamics of a Diffusing Interface

\section{FUNDING NUMBERS}

$$
\text { WU-101-53-00-00 }
$$

8. PERFORMING ORGANIZATION REPORT NUMBER

$E-12810$

10. SPONSORING/MONITORING AGENCY REPORT NUMBER

NASA TM-2001-210956

AIAA-2001-1134

\section{SUPPLEMENTARY NOTES}

Prepared for the 39th Aerospace Sciences Meeting and Exhibit sponsored by the American Institute of Aeronautics and Astronautics, Reno, Nevada, January 8-11, 2001. Responsible person, Walter M.B. Duval, organization code $6712,216-433-5023$.

\section{2a. DISTRIBUTION/AVAILABILITY STATEMENT}

Unclassified - Unlimited

Subject Categories: 29 and 34

Distribution: Nonstandard

Available electronically at hitpi//gltrs.grc.nasa.gov/GLTRS

This publication is available from the NASA Center for AeroSpace Information, 301-621-0390.

\section{ABSTRACT (Maximum 200 words)}

Excitation of two miscible viscous liquids inside a bounded enclosure in a microgravity environment has shown the evolution of quasi-stationary waves of various modes for a range of parameters. We examine computationally the nonlinear dynamics of the system as the interface breakup and bifurcates to resonance structures typified by the Rayleigh-Taylor instability mechanism. Results show that when the mean steady field is much smaller than the amplitude of the sinusoidal excitation, the system behaves linearly, and growth of quasi-stationary waves occurs through the Kelvin-Helmholtz instability mechanism. However, as the amplitude of excitation increases, nonlinearity occurs through subharmonic bifurcation prior to broadband chaos.

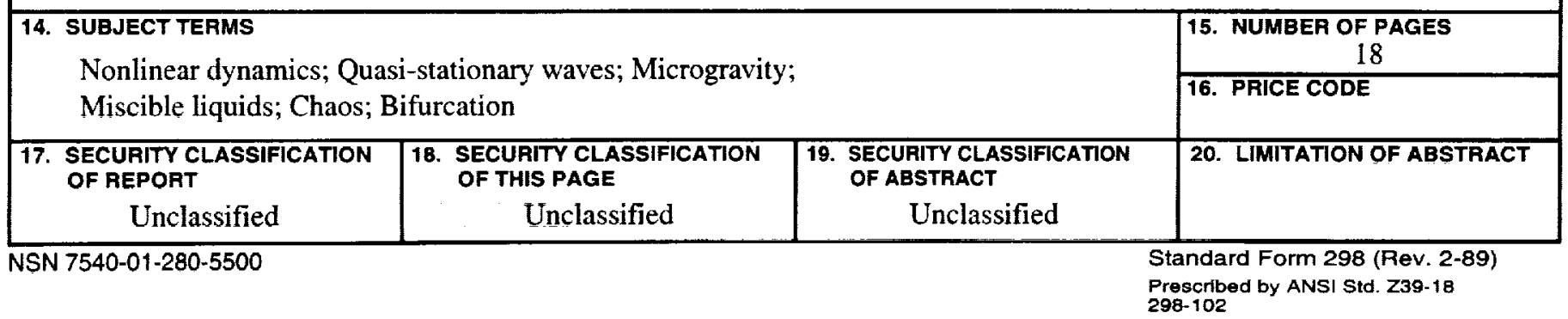

